\title{
Clinical spectrum and presentation of patients with absent contractility
}

\author{
Balazs Kovacs ${ }^{a}$, Takahiro Masuda ${ }^{a, b}$, Ross M. Bremnera, Sumeet K. Mittal ${ }^{a, b}$
}

Norton Thoracic Institute, St. Joseph's Hospital and Medical Center, Phoenix, AZ, USA; Creighton University School of Medicine, Phoenix Regional Campus, Phoenix, AZ, USA

Abstract

\section{Introduction}

Primary absent contractility is an uncommon finding on high-resolution manometry (HRM), and this condition has been associated with autoimmune disorders. An aperistaltic

${ }^{a}$ Norton Thoracic Institute, St. Joseph's Hospital and Medical Center (Balazs Kovacs, Takahiro Masuda, Ross M. Bremner, Sumeet K. Mittal); ${ }^{\mathrm{b} C}$ reighton University School of Medicine, Phoenix Regional Campus (Takahiro Masuda, Sumeet K. Mittal), Phoenix, AZ, USA

Conflict of Interest: None

Correspondence to: Sumeet K. Mittal, MD, Norton Thoracic Institute, St. Joseph's Hospital and Medical Center, 500 W. Thomas Rd., Suite 500, Phoenix, AZ 85013, USA, e-mail: Sumeet.Mittal@DignityHealth.org

Received 18 July 2020; accepted 18 November 2020; published online 25 January 2021

DOI: https://doi.org/10.20524/aog.2021.0583 esophagus was first described in the conventional manometric era, with somewhat different characteristics. The classification of absent contractility has had multiple iterations as esophageal motility studies have evolved, and the currently accepted definition of absent contractility is described by the Chicago Classification v.3.0 [1]. The definition of absent contractility has evolved with the advancement of esophageal manometry. For HRM, the first version of the Chicago classification defined absent contractility as "no continuous pressure domain above an isobaric contour of $30 \mathrm{mmHg}$ in the distal esophageal segment in any swallow; and scleroderma pattern: no continuous pressure domain above an isobaric contour of $30 \mathrm{mmHg}$ in the distal esophageal segment in any swallow and a mean lower esophageal sphincter (LES) pressure (LESP) $<10 \mathrm{mmHg}$ " [2]. The second version of the Chicago classification defined it as "normal mean integrated relaxation pressure (IRP), 100\% of swallows with failed peristalsis" [3], similar to the final version of Chicago classification v.3.0. However, the third version (i.e., 
the current version) changed the definition of failed peristalsis, from "minimal $(<3 \mathrm{~cm})$ integrity of the $20 \mathrm{mmHg}$ isobaric contour in the distal esophagus" to "distal contractile integral (DCI) <100," which is easier to interpret [1]. Each update reflects stricter, more specific criteria. However, this means that comparisons and discussions of patients deemed "aperistaltic" by different classifications might be fraught with errors, but given the sparse literature of this topic, there is no alternative. An aperistaltic esophagus noted in type I achalasia is a very different clinical entity than primary absent contractility. The causative and associated factors of this condition are not well understood, and absent contractility is associated with challenging clinical scenarios.

Some have pointed out a possible association between absent contractility and systemic sclerosis (SSc), dysphagia, and severe gastroesophageal reflux disease (GERD); limited data describe patients who have absent contractility in the absence of either SSc or GERD. Recently, some have reported on the association of absent contractility with systemic disorders other than SSc and GERD [4]. The goal of this study was to describe and explore the clinical spectrum and presentation of patients diagnosed with absent contractility at a tertiary referral center.

\section{Patients and methods}

\section{Patient population}

This study was approved by our center's Institutional Review Board. At our center, we enter all patients who undergo esophageal function testing into a prospectively maintained database. For this study, we queried the database to identify those who underwent HRM between August 2016 and September 2018 and were diagnosed with absent contractility. We excluded patients who had: 1) previous foregut surgery or endoscopic intervention; 2) a poor HRM study or missing medical information; or 3 ) other clinical testing consistent with achalasia or history of achalasia.

\section{HRM}

HRM was performed using a 36-channel probe with circumferential solid-state pressure transducers at $1-\mathrm{cm}$ intervals (Medtronic, Minneapolis, MN). HRM studies were reanalyzed using Manoview software version 3.0.1 (Medtronic) by a single author (BK), who was blinded to the outcomes of the other testing results and clinical findings. The pressure topography of $105-\mathrm{mL}$ water swallows was assessed using Chicago Classification v3.0 [1]. According to this classification, "absent peristalsis" (i.e., absent contractility) was defined as $100 \%$ failed water swallows, DCI $<100$, and IRP4s $<15 \mathrm{mmHg}$. DCI and IRP4s were collected and calculated by Manoview software version 3.0.1 in concordance with Chicago classification v.3.0 [1]. LESP was calculated as the average pressure during both inspiration and expiration in the eSleeve window (respiratory mean) referred to gastric pressure by software [5]. Fig. 1 shows the appearance of absent contractility on HRM. No provocative maneuvers (e.g., multiple rapid swallows) were conducted.

\section{Ambulatory pH testing}

Ambulatory $\mathrm{pH}$ testing was carried out using either a dual-channel catheter $\mathrm{pH}$ testing system (Digitrapper $400 \mathrm{pH}$, Medtronic) or a wireless single-channel $\mathrm{pH}$ testing probe (Bravo, Medtronic). The catheter-based $\mathrm{pH}$ probe was passed transnasally and positioned $5 \mathrm{~cm}$ above the upper border of the manometrically defined LES. The capsule-based system was passed transorally and positioned $6 \mathrm{~cm}$ above the gastroesophageal junction under endoscopic guidance. In accordance with the Lyon consensus [6], the distal esophageal AET was calculated as the percentage of time $\mathrm{pH}$ was $<4.0$, measured over the course of 2 days, for the capsule-based system. For the catheter-based system, distal esophageal AET was calculated as the percentage of time $\mathrm{pH}$ was $<4.0$, measured on the distal esophageal $\mathrm{pH}$ probe, $5 \mathrm{~cm}$ above the LES. All patients were required to keep track of supine periods, eating, drinking and gastrointestinal symptoms; these logs were compared to the electronic diary, and the diagrams were corrected if necessary. All measurements had an analysis time of at least $18 \mathrm{~h}$. All pH testing was conducted while the patients were not taking any acid suppression medications.

\section{Symptom questionnaire}

All patients who underwent functional esophageal testing were asked to complete a standardized foregut symptom questionnaire (Appendix 1). The questionnaire assessed the severity of foregut symptoms a patient may experience, such as heartburn, dysphagia, chest pain, regurgitation and abdominal bloating. Each symptom was scored on a scale of 0-4 (Grade 0: none; Grade 1: mild, less than once a week; Grade 2: moderate, several times a week; Grade 3: severe, daily, affecting the patient's lifestyle; and Grade 4: very severe, always present, markedly affecting the patient's lifestyle).

Patients' detailed medical history was retrieved from electronic medical records, as were the clinical results of gastroduodenoscopy, histopathology and barium swallow tests. Descriptive statistics were applied to the data retrieved. Continuous variables were described as mean \pm standard deviation or median (interquartile range [IQR]).

\section{Results}

In total, 2068 patients underwent HRM at our institution between August 2016 and September 2018. Of these, 112 (5.4\%) were diagnosed with absent esophageal contractility. Forty-six patients were excluded: 25 patients were referred from 


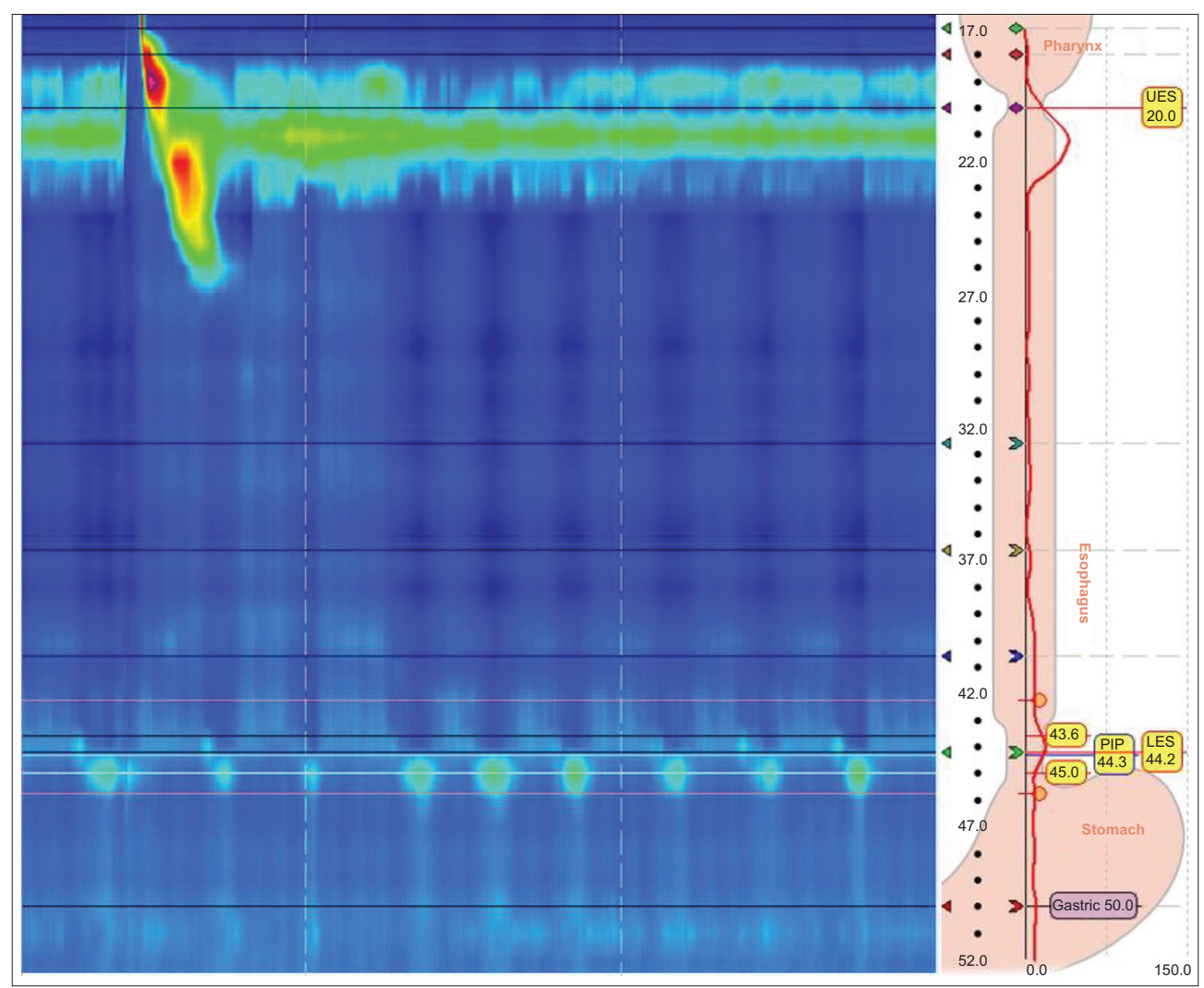

Figure 1 Typical representation of absent contractility on high-resolution manometry PIP, pressure inversion point; LES, lower esophageal sphincter

an outside facility solely for testing or because they had poor quality studies in which proper gastric reference pressure could not be obtained because of artifacts or suspected coiling; 11 were excluded because they had undergone previous foregut surgery; and 10 were excluded because of a history of achalasia or findings consistent with achalasia on clinical testing other than HRM. This left 66 patients who met the inclusion criteria and formed the cohort of this study. Of these, 38 patients (57.6\%) were women, and the mean age of the cohort was $56.6 \pm 13.86$ years. The mean body mass index was $26.37 \pm 5.7 \mathrm{~kg} / \mathrm{m}^{2}$.

The primary complaint indicating a diagnostic workup was heartburn or regurgitation in $54.5 \%(36 / 66)$ of the patients. Nearly half of the patients in this study $(29 / 66,43.9 \%)$ underwent HRM as part of an evaluation for lung transplant. Fifty-nine patients had a detailed medical history available, and $22 / 59$ patients $(37.3 \%)$ had a history of autoimmune, immune-mediated, or myopathic diseases, while 29/59 patients (49.2\%) had some form of interstitial lung disease (Table 1). Of the patients with a history of autoimmune disease, 11 had scleroderma (10 scleroderma with SSc) and 5 patients had idiopathic pulmonary fibrosis with signs of a localized or systemic autoimmune problem. There were no cases of obstructive lung disease in the cohort. Fourteen of the 66 patients had diabetes mellitus, and all but 1 of these 14 were in the lung transplant evaluation subgroup.

In addition to the HRM studies, 51/66 patients completed the symptom questionnaire. Of these, 42 patients $(82.4 \%)$
Table 1 Autoimmune and myopathic diseases and interstitial lung disease in 66 patients

\begin{tabular}{lc}
\hline Disorder & $\mathrm{n}(\%)$ \\
\hline Not available & $7(10.6)$ \\
No history of ILD, AI or MP & $13(19.7)$ \\
Interstitial lung disease & \\
Unspecified ILD & $8(12.1)$ \\
Idiopathic pulmonary fibrosis & $9(13.6)$ \\
IPF with autoimmune features & $5(7.6)$ \\
NSIP & $3(4.5)$ \\
Other rare lung disease & $4(6.1)$ \\
Autoimmune and myopathic diseases & \\
Scleroderma & $11(16.7)$ \\
Fibromyalgia & $3(4.5)$ \\
Muscular dystrophy & $1(1.5)$ \\
Mixed connective tissue disease & $2(3.0)$ \\
\hline
\end{tabular}

${ }^{*}$ Combined pulmonary fibrosis and emphysema, cystic fibrosis, alveolar microlithiasis, hypersensitivity pneumonitis

ILD, interstitial lung disease; AI, autoimmune disease; MP, myopathic disease; IPF, idiopathic pulmonary fibrosis; NSIP, nonspecific interstitial pneumonia

reported some degree of heartburn, and 17/51 patients (33.3\%) reported experiencing severe heartburn (Grade 3 or higher); $24 / 51$ patients $(47.1 \%)$ reported dysphagia; $11 / 51$ patients 
(21.6\%) reported experiencing severe dysphagia (Grade 3 or higher); 23/51 patients (45.1\%) reported both heartburn and dysphagia; and $9 / 51$ patients (17.6\%) reported experiencing severe levels of each. Fig. 2 contains additional detail on some of the more commonly reported symptoms. Forty-two of the 51 patients reported additional symptoms, including change of voice $(25 / 51,49.0 \%)$, recurrent cough $(25 / 51,49.0 \%)$, night cough $(21 / 51,41.2 \%)$, nausea and vomiting $(17 / 51,33.3 \%)$, and recurrent pneumonia or bronchitis $(9 / 51,17.6 \%)$. Reported symptom characteristics did not differ in the lung transplant evaluation subgroup.

In terms of HRM findings, $12 / 66$ patients (18.2\%) had hiatal hernia on HRM. The median IRP4s was $4.8 \mathrm{mmHg}$, and the average LESP was $21 \mathrm{mmHg}$. Thirty-seven patients underwent ambulatory $\mathrm{pH}$ monitoring within 2 weeks of HRM (6 via a wireless testing method); of these, 23 patients $(62.2 \%)$ had pathological distal esophageal exposure (AET $>6 \%$ ). The mean distal esophageal AET was $8.7 \pm 7.4 \%$ and the median distal esophageal AET was 7.4\% (IQR 9.9\%). The mean reflux time was much more pronounced in the upright position than in the supine position $(10.4 \pm 10 \%$ upright vs. $5.3 \pm 8.3 \%$ supine; Fig. 3). The total numbers of reflux events were also compared and no difference was found.

Fifty patients had esophagogastroduodenoscopy results available, 45 of which also had biopsies. Esophageal candidiasis was confirmed in $4 / 45$ patients (8.8\%). Pathologically confirmed esophagitis was present in $26 / 45$ patients $(57.7 \%$ ).

Fifty-two patients had an esophagram available. Seventeen of these patients (32.7\%) had delayed esophageal emptying, $11(21.2 \%)$ were noted to have dilation of the esophagus (IRP in this subgroup was under $11 \mathrm{mmHg}$; none of these patients showed characteristics of achalasia on HRM), and 2 patients (3.8\%) were also diagnosed with Zenker's diverticulum.

\section{Discussion}

Absent contractility on esophageal manometry was first associated with scleroderma, known to affect esophageal contractility. The prevalence of absent contractility in patients with SSc is reported to be as high as 55\% [7]. However, as HRM is becoming more commonly used as a diagnostic tool in patients with functional esophageal diseases, we are facing

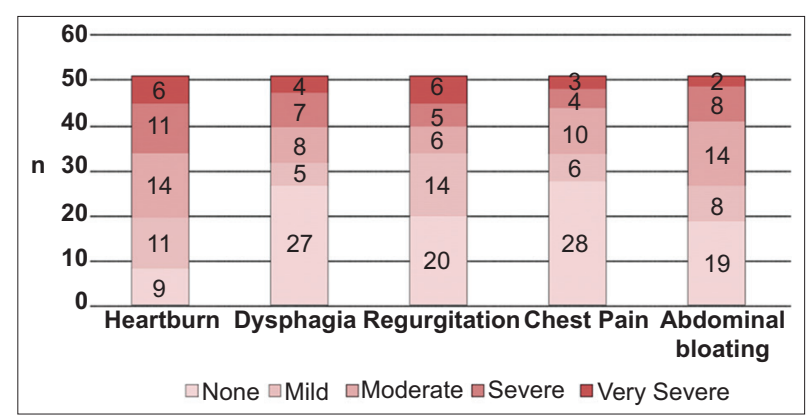

Figure 2 Distribution of 5 symptoms assessed by questionnaire more cases of absent contractility in patients without a clinical history of scleroderma. Although only a few publications address the absence of contractility outside the scope of scleroderma, it seems that a number of other autoimmune disorders, myopathic diseases and non-immunologic factors may be linked to absent contractility $[4,8,9]$. Of the patients in our cohort, $37.3 \%$ had a history of autoimmune, immunemediated or myopathic diseases of various origin, while only $15.2 \%$ had scleroderma. This indicates that a wider array of systemic processes could have a causative association with absent contractility. Laique et al reported that as many as $80 \%$ of their patient populations were affected by systemic autoimmune rheumatologic disease [4].

Gasper et al noted that esophageal dysmotility, including absent contractility, is common in lung transplant candidates diagnosed with connective tissue disease. Dysmotilityassociated reflux and aspiration have since been found to drastically affect the long-term outcomes of lung transplantation [9]. Esophageal dysmotility might also play a role in the evolution of some lung diseases [10]. In our cohort, 29 of 66 patients were tested while being evaluated for potential lung transplantation, and we plan a further follow up of these patients in another study. We have previously reported that esophageal motility improves in a subset of patients with interstitial lung disease post-transplant [10]. This would indicate that esophageal dysmotility in patients with interstitial lung disease is at least partly due to lateral traction from fibrosis and deranged pulmonary dynamics [11].

Similar to our observations, most reports agree that GERD is a primary problem of patients with absent contractility, and GERD symptoms such as heartburn and regurgitation are experienced by many patients with absent contractility [4]. Smout et al [8] observed a high prevalence of reflux and Barrett's esophagus in this patient population. Dysphagia is present in a number of patients who do not have objective signs of outflow problems or bolus-clearance issues $[8,9]$. Denaxas hypothesized that some of this dysphagia might be a result of Candida esophagitis, also more prevalent in patients with absent contractility [7]. As demonstrated in our cohort, dilated esophagus is seen in advanced-stage esophageal disease even without outflow obstruction, such as in "burned out" reflux and scleroderma [12].

No definitive therapy is available for patients diagnosed with absent contractility. However, our experience with lung transplant patients suggests that restoration of normal intrathoracic aerodynamics improves esophageal motility in certain patients with restrictive pulmonary disease [11]. For medical treatment, both metoclopramide and erythromycin may increase LESP, while cisapride seems to increase LESP and distal esophageal body peristalsis. All 3 of these medications have been used in clinical practice, but their effectiveness has not been clearly reported [8]. A study of 22 patients with symptomatic SSc (under proton pump inhibitors) found that buspirone effectively ameliorated symptoms and significantly improved objective parameters such as LESP [7].

Watson et al proposed that partial fundoplication for GERD control in patients with absent contractility might be adequate and does not pose as high a threat of dysphagia; however, most centers do not perform fundoplication in patients who have 


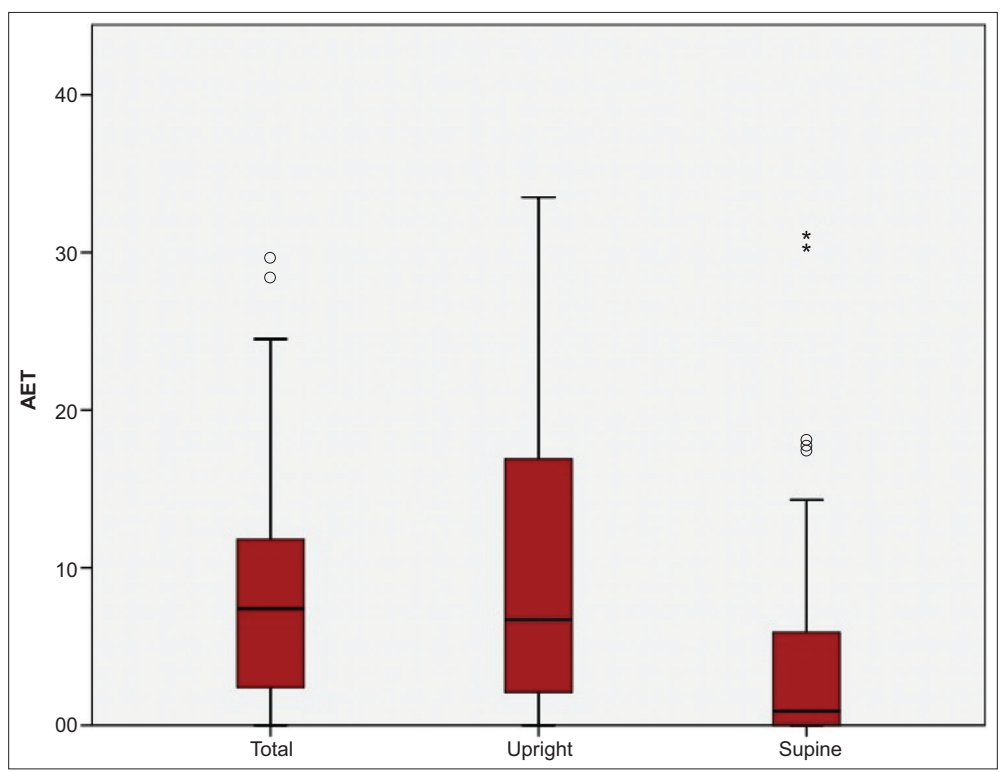

Figure 3 Box plot of distal esophageal acid exposure time (AET) of the cohort

absent peristalsis $[4,13]$. Hiatal hernia repair might also improve dysphagia in patients who have both hiatal hernia and absent contractility [8]. Kent reported that Roux-en-Y gastric bypass as an antireflux measure is superior to partial fundoplication and has less morbidity than esophageal resection for symptom control in patients with scleroderma [14].

Although most of our data were collected prospectively, our study was limited by its retrospective design. A longitudinal study and direct comparison with a healthy control group would greatly benefit our understanding of this rare clinical entity. Even though the results of conventional and wireless $\mathrm{pH}$ metrics were combined, the clearly pathological results (AET $>6 \%$ ) of patients tested with a wireless probe system mitigate the inaccuracies that may arise from the different normative values of these modalities.

The inclusion of provocative testing (e.g., multiple rapid swallows or standardized solid test meal) in our HRM testing protocol might be beneficial in a subset of these patients, as normal IRP on standard testing might hide achalasialike disease. Further investigations, including impedance planimetry and solid bolus on timed barium swallow, should be considered for patients with absent contractility and pronounced dysphagia $[15,16]$.

Absent contractility is rare and can occur concurrently with a wide array of diseases; the usual clinical presentation is reflux, dysphagia or both. A large number of patients in our study were undergoing lung transplant evaluation; this could be due to a causative underlying disease process, or it may be reflective of the effects of deranged pulmonary dynamics on esophageal motility.

\section{Acknowledgment}

The authors are grateful to Clare Sonntag, who provided editorial assistance for this manuscript.

\section{Summary Box}

\section{What is already known:}

- Scleroderma is linked to absent contractility on high-resolution esophageal manometry

- Gastroesophageal reflux disease is a primary problem in patients with absent contractility

\section{What the new findings are:}

- A number of other autoimmune disorders, outside the scope of scleroderma, might be linked to absent contractility on high-resolution esophageal manometry

\section{References}

1. Kahrilas PJ, Bredenoord AJ, Fox M, et al; International High Resolution Manometry Working Group. The Chicago Classification of esophageal motility disorders, v3.0. Neurogastroenterol Motil 2015;27:160-174.

2. Kahrilas PJ, Ghosh SK, Pandolfino JE. Esophageal motility disorders in terms of pressure topography: the Chicago Classification. J Clin Gastroenterol 2008;42:627-635.

3. Bredenoord AJ, Fox M, Kahrilas PJ, Pandolfino JE, Schwizer W, Smout AJ; International High Resolution Manometry Working Group. Chicago classification criteria of esophageal motility disorders defined in high resolution esophageal pressure topography. Neurogastroenterol Motil 2012;24 Suppl 1:57-65.

4. Laique S, Singh T, Dornblaser D, et al. Clinical characteristics and associated systemic diseases in patients with esophageal "absent contractility" - a clinical algorithm. J Clin Gastroenterol 2019;53:184-190. 
5. Niebisch S, Wilshire CL, Peters JH. Systematic analysis of esophageal pressure topography in high-resolution manometry of 68 normal volunteers. Dis Esophagus 2013;26:651-660.

6. Gyawali CP, Kahrilas PJ, Savarino E, et al. Modern diagnosis of GERD: the Lyon Consensus. Gut 2018;67:1351-1362.

7. Denaxas K, Ladas SD, Karamanolis GP. Evaluation and management of esophageal manifestations in systemic sclerosis. Ann Gastroenterol 2018;31:165-170.

8. Smout A, Fox M. Weak and absent peristalsis. Neurogastroenterol Motil 2012;24 Suppl 1:40-47.

9. Gasper WJ, Sweet MP, Golden JA, et al. Lung transplantation in patients with connective tissue disorders and esophageal dysmotility. Dis Esophagus 2008;21:650-655.

10. Fisichella PM, Reder NP, Gagermeier J, Kovacs EJ. Usefulness of $\mathrm{pH}$ monitoring in predicting the survival status of patients with scleroderma awaiting lung transplantation. J Surg Res 2014;189:232-237.

11. Masuda T, Mittal SK, Kovács B, et al. Foregut function before and after lung transplant. J Thorac Cardiovasc Surg 2019;158:619-629.
12. Khandelwal N, Chowdhury V, Gupta AK, Srivastava DN. Diagnostic radiology: gastrointestinal and hepatobiliary imaging. $3^{\text {rd }}$ edn, Jaypee Brothers Medical Publishers (P) Ltd.; 2009.

13. Watson DI, Jamieson GG, Bessell JR, Devitt PG. Laparoscopic fundoplication in patients with an aperistaltic esophagus and gastroesophageal reflux. Dis Esophagus 2006;19:94-98.

14. Kent MS, Luketich JD, Irshad K, et al. Comparison of surgical approaches to recalcitrant gastroesophageal reflux disease in the patient with scleroderma. Ann Thorac Surg 2007;84:1710-1715; discussion 1715-1716.

15. Hollenstein M, Thwaites P, Bütikofer S, et al. Pharyngeal swallowing and oesophageal motility during a solid meal test: a prospective study in healthy volunteers and patients with major motility disorders. Lancet Gastroenterol Hepatol 2017;2:644-653.

16. Ang D, Misselwitz B, Hollenstein M, et al. Diagnostic yield of highresolution manometry with a solid test meal for clinically relevant, symptomatic oesophageal motility disorders: serial diagnostic study. Lancet Gastroenterol Hepatol 2017;2:654-661. 


\section{Supplementary material}

\section{Appendix 1 Symptom questionnaire}

1. Heartburn

0, Never:

1, Mild: Occasionally (less than once a week) It can be ignored if I don't think about it

2, Moderate: Sometimes (several times a week). It cannot be ignored, but does not affect my lifestyle. 3, Severe: Often (daily). It affects my lifestyle.

4, Always (with every meal). It markedly affects my lifestyle.

2. Regurgitation

0, Never:

1, Mild: Occasionally (less than once a week) It can be ignored if I don't think about it

2, Moderate: Sometimes (several times a week). It cannot be ignored, but does not affect my lifestyle. 3, Severe: Often (daily). It affects my lifestyle.

4, Always (with every meal). It markedly affects my lifestyle.

3. Difficulty swallowing:

0, Never:

1, Mild: Occasionally (less than once a week) It can be ignored if I don't think about it

2, Moderate: Sometimes (several times a week). It cannot be ignored, but does not affect my lifestyle. 3, Severe: Often (daily). It affects my lifestyle.

4, Always (with every meal). It markedly affects my lifestyle.

4. Chest pain:

0, Never:

1, Mild: Occasionally (less than once a week) It can be ignored if I don't think about it

2, Moderate: Sometimes (several times a week). It cannot be ignored, but does not affect my lifestyle.

3, Severe: Often (daily). It affects my lifestyle.

4, Always (with every meal). It markedly affects my lifestyle. 